\title{
A prospective study to evaluate the efficacy and safety of oral acetyl-L-carnitine for the treatment of chemotherapy-induced peripheral neuropathy
}

\author{
YUANJUE SUN ${ }^{1}$, YONGQIAN SHU ${ }^{2}$, BAORUI LIU ${ }^{3}$, PING LIU ${ }^{2}$, CHANGPING WU ${ }^{4}$, \\ RONGSHENG ZHENG ${ }^{5}$, XIAOHUA ZHANG ${ }^{6}$, ZHIXIANG ZHUANG ${ }^{7}$, YONGCHUAN DENG $^{8}$, \\ LEIZHEN ZHENG $^{9}$, QING XU ${ }^{10}$, BIN JIANG $^{11}$, XUENONG OUYANG ${ }^{12}$, JIANFEI GAO ${ }^{13}$, \\ NONG XU ${ }^{14}$, XIAOYI LI ${ }^{15}$, SU JIANG ${ }^{16}$, CHAOFAN LIANG ${ }^{15}$ and YANG YAO ${ }^{1}$
}

${ }^{1}$ Oncology Department, The 6th People's Hospital Affiliated to Shanghai Jiao Tong University, Shanghai 200233;

${ }^{2}$ Oncology Department, The First Affiliated Hospital of Nanjing Medical University, Nanjing, Jiangsu 210029;

${ }^{3}$ Oncology Department, Nanjing Drum Tower Hospital, The Affiliated Hospital of Nanjing University Medical School, Nanjing, Jiangsu 210008; ${ }^{4}$ Oncology Department, The First People's Hospital of Changzhou, Changzhou, Jiangsu 213003;

${ }^{5}$ Oncology Department, The First Affiliated Hospital of BengBu Medical College, Bengbu, Anhui 233004;

${ }^{6}$ Surgical Oncology Department, The First Affiliated Hospital of Wenzhou Medical College, Wenzhou, Zhejiang 325000;

${ }^{7}$ Oncology Department, The Second Affiliated Hospital of Soochow University, Suzhou, Jiangsu 215004;

${ }^{8}$ Surgical Oncology Department, The Second Affiliated Hospital of Zhejiang University School of Medicine, Hangzhou,

Zhejiang 310009; ${ }^{9}$ Oncology Department, Xinhua Hospital, Shanghai Jiaotong University School of Medicine,

Shanghai 200092; ${ }^{10}$ Oncology Department, Shanghai Tenth People's Hospital, Shanghai 200072; ${ }^{11}$ Oncology Department,

Third People's Hospital Affiliated to Shanghai Jiao Tong University School of Medicine, Shanghai 201999;

${ }^{12}$ Oncology Department, Fuzhou General Hospital of Nanjing Military Command of Chinese PLA, Fuzhou, Fujian 350025;

${ }^{13}$ Cancer Hematology Department, Wuhan General Hospital of Guangzhou Military Command of Chinese PLA, Wuhan, Hubei 510000; ${ }^{14}$ Oncology Department, The First Affiliated Hospital of Zhejiang University School of Medicine, Hangzhou, Zhejiang 310003; ${ }^{15}$ Lee's Pharmaceutical (Hong Kong) Ltd., Hong Kong;

${ }^{16}$ Zhaoke Pharmaceutical (Heifei) Co. Ltd., Hefei, Anhui 230088, P.R. China

Received May 6, 2015; Accepted July 5, 2016

DOI: $10.3892 / \mathrm{etm} .2016 .3871$

\begin{abstract}
The present study aimed to evaluate the efficacy and safety of acetyl-L-carnitine (ALC) for the treatment of chemotherapy-induced peripheral neuropathy (CIPN). The study was carried out as a prospective, randomized, double-blind, placebo-controlled and paralleled clinical study. A total of 239 patients with CIPN were selected as the study subjects. Of the 239 subjects, 118 subjects received $3 \mathrm{~g} / \mathrm{day}$ ALC orally for 8 weeks and 121 received a placebo. The primary endpoint was improvement of peripheral neuropathy
\end{abstract}

Correspondence to: Dr Yang Yao, Oncology Department, The 6th People's Hospital Affiliated to Shanghai Jiao Tong University, 600 Yishan Road, Shanghai 200233, P.R. China

E-mail: yangyao201501@sina.com

Key words: acetyl-L-carnitine, chemotherapy-induced peripheral neuropathy, cancer-associated fatigue, adverse events, sensory neuropathy by at least one grade. Patient status was assessed at week 4,8 and 12 after enrollment into the study. In both the full analysis set (FAS) and the per-protocol set (PPS), peripheral sensory neuropathy was significantly ameliorated in the ALC group with 50.5 and $51.6 \%$ patients meeting the primary endpoint at week 8 , compared with 24.1 and $23.1 \%$ of patients in the placebo group $(\mathrm{P}<0.001$ in both sets). Secondary endpoints, such as the nerve electrophysiological examination and the Karnofsky physical score were also significantly improved in patients receiving ALC treatment, as compared with the placebo group (FAS, $\mathrm{P}=0.0463$ and $\mathrm{P}=0.022$; $\mathrm{PPS}, \mathrm{P}=0.0076$ and $\mathrm{P}=0.0064$, respectively). Cancer-associated fatigue was significantly alleviated following ALC treatment in the PPS $(\mathrm{P}=0.0135)$. In the safety analysis set, the difference in adverse events incidence between the two groups was not statistically significant $(\mathrm{P}=0.3903)$. There were only two severe adverse events in the ALC group, which were not associated with the effect of ALC. In conclusion, the results of the present study demonstrated that in Chinese patients with cancer, oral administration of ALC is effective at ameliorating peripheral sensory neuropathy induced by chemotherapy, as well as reducing of cancer-associated fatigue and improving physical conditions. 


\section{Introduction}

Chemotherapy-induced peripheral neuropathy (CIPN) is a common, dose-limiting adverse drug reaction in cancer treatment (1), which primarily presents as varying degrees of motor and sensory deficits, as well as autonomic dysfunction. Currently, paclitaxel, cisplatin, and vinblastine are the most commonly prescribed anti-cancer chemotherapy drugs (2). Unfortunately, these drugs all produce treatment-limiting peripheral neuropathy, for which there is no reliable clinical intervention. The primary treatment of CIPN is to reduce the chemotherapy dose and to extend the interval between treatments, or cease treatment completely (3). However, this is not an optimal choice for the long-term prognosis of the patient.

Acetyl-L-carnitine (ALC) is a nutrient supplement with the ability to stimulate the expression of nerve growth factor receptor, strengthen the tubulin of nerve cells and prevent cytoskeletal damage and cystic nerve fibrosis, as well as improve sensory nerve conduction $(4,5)$. In addition, numerous basic and clinical studies have demonstrated that ALC alleviates CIPN without reducing the antitumor drug activity (6-8).

Sigma Tau Pharmaceuticals, Inc. developed levocarnitine acetate hydrochloride gastro-resistant tablets (Nicetile ${ }^{\circledR}$ ), which is an oral drug that first appeared on the Italian market in July 1984, with peripheral nerve or nerve root mechanisms of action and inflammatory injury as the registered indication. However, the effects of Nicetile ${ }^{\circledR}$ in Chinese individuals with CIPN remains to be elucidated. The aim of the present study was to investigate the efficacy and safety of levocarnitine acetate hydrochloride gastro-resistant tablets on CIPN in a large Chinese population.

\section{Materials and methods}

Study design and approval. This study was a multicenter, randomized, double-blind, and placebo-controlled phase II clinical trial. It was approved by the Chinese State Food and Drug Administration (approval no. 2007L03540). The clinical trial registration number is NCT01526564. The clinical study was carried out in accordance with The Code of Ethics of the World Medical Association (Declaration of Helsinki) for experiments involving humans. In addition, informed consent was obtained from all participants involved in this study.

Eligible patients were aged 18-75 years without gender limitation. Eligibility criteria included: Grade $\geq 3$ neuropathy, as determined by NCI-CTC criteria version 3.0 (9), while receiving paclitaxel, cisplatin or vinblastine treatment, and/or grade $\geq 2$ neuropathy persisting for at least one month after the discontinuation of either drug, and neurotoxicity for $<6$ months; at least one abnormality on electrophysiological examination; Karnofsky physical score (KPS) of $\geq 60$; absolute neutrophil count of $\geq 1.5 \times 10^{9} / 1$, hemoglobin count of $\geq 80 \mathrm{~g} / 1$, platelet count of $\geq 75 \times 10^{9} / 1$, total bilirubin counts of 1.5 -fold less than normal value, glutamic-pyruvic transaminase (GPT/ALT) and glutamic-oxalacetic transaminease (GOT/AST) no more than 2.5-fold greater than the normal value; normal blood urea nitrogen, serum creatinine and electrocardiogram (ECG) findings. During the study, the use of steroids, analgesic or neuroprotectant drugs was not permitted. Patients were enrolled after providing written informed consent.

Exclusion criteria included: Neuropathy caused by other antineoplastic treatment except paclitaxel, cisplatin or vinblastine; pre-existing diabetes mellitus and/or neuropathy caused by vitamin deficiency, infection, trauma, poisoning, oppression, ischemia, metabolic disorders; genetic neuropathy and/or peripheral sensory nerve dysfunction due to central nervous system lesions; use of other drug therapy for neuropathy in the last 30 days (such as nerve growth factor, amifostine reduced glutathione, vitamin E or B, glucocorticoids, ethosuximide, carbamazepine, gabapentin, sodium thiosulfate, glutamic acid, lamotrigine, $\alpha$-fatty acid, lithium salt, lithium salt or magnesium salt); participation in other clinical trials in the past 30 days; out of control clinical problems (such as serious mental, nerve, cardiovascular and/or respiratory system disease); pregnant or lactating women; and poor compliance.

During the trial, patients were withdrawn if serious adverse events occurred, and/or the patient became pregnant.

Patients. A total of 240 patients met the criteria and were recruited for the present study at Shanghai Changzheng Hospital (Shanghai, China) between September 4, 2010 and November 7, 2013. All patients were treated with at least one type of taxoid, either satraplatin $\left(80 \mathrm{mg} / \mathrm{m}^{2}\right.$; GPC Biotech, Munich, Germany) or vincristine $\left(0.05 \mathrm{mg} / \mathrm{kg}^{2}\right.$; Pharmachemic Hisun, Zhejiang, China). Their peripheral sensory neuropathy grading following chemotherapy was $\geq$ grade 3 or 2 , lasted for $\geq 1$ month, and the course of neuropathy was $\leq 6$ months. The patient either no longer required chemotherapy or did not choose to undergo chemotherapy. Each patient was administered one ALC hydrochloride enteric-coated tablet (oral administration; $500 \mathrm{mg} / \mathrm{tablet}, 2$ tablets/time) three times a day for eight consecutive weeks. The patients of the control group received a placebo (lactose, $500 \mathrm{mg} /$ tablet, 2 tablets/ time) in the same manner. Patients were instructed that the interval between doses should be at $\geq 4 \mathrm{~h}$ and were monitored at week 4,8 and 12. Since drug treatment ceased after week 8 , the results obtained after week 12 represented the long-term effects of ALC intervention.

Safety analysis set (SS) was an analysis in those who received at least one dose of the investigational drug and safety valuation. Full analysis set (FAS) was analysis in which patients are included in the group to which they were randomized irrespective of compliance, administrative errors (such as error in eligibility), or other protocol deviations. Per protocol set (PPS) was an analysis in which patients are included in the group corresponding to the treatment they actually received. Patient compliance and "switchovers" were considered in the analysis.

Primary endpoint. The primary endpoint of the study was to demonstrate an improvement of $\geq 1$ grade in neurotoxicity, as determined by NCI-CTC criteria (version 3.0) at week 8 of ALC administration (9).

Secondary endpoints. Prior to ALC administration at 4, 8 and 12 weeks, electrophysiological examinations were carried out at each time point, which included examination of the nerves (handedness, median nerve, ulnar nerve, common peroneal 
nerve, tibial nerve, superficial peroneal nerve and sural nerve). Three neural electricity experts compared the electrophysiology examination results at the 8th week (including nerve conductive velocity, latency period and amplitude) to the results obtained prior to ALC administration. The unified curative effect standard to centralized assess was followed and the evaluation results were recorded and signed by the experts. The results were categorized into improved, effective improved and no change, based on the efficient rate which was calculated by the following formula: Efficient rate $=($ Number of patients that improved + Number of effective improved patients) / (All patients - Number of patients that could not be evaluated) x $100 \%$.

Evaluation of safety and efficacy. The case histories of the patients, previous chemotherapy information, and disease and treatment histories were recorded during screening. Prior to 4,8 , and 12 weeks of testing, all patients underwent baseline assessment, which included comprehensive physical examinations, laboratory examinations, and electrophysiological and 12-lead ECG examination. The physical examinations included the measurement of vital signs (temperature, pulse, respiration and blood pressure), the grading of neurotoxicity (9), cancer-associated fatigue classification (10) and KPS assessment (11). Laboratory examination included a routine blood panel, liver and kidney function (12) and fasting glucose measurement (13). Electrophysiology examination was performed as described and the results were categorized into improved, effective improved and no change. During the study, all disease factors, drug combinations and adverse events were recorded in the case report form (CRF).

Nerve conductive velocity ( $N C V$ ). $\mathrm{NCV}$ was examined to evaluate the changes prior to ALC administration and after 8 weeks of ALC administration. Treatment was defined as effective if the NCV was improved after ALC administration, whereas, it was defined as ineffective if the NCV was reduced or unchanged. Effective analysis was conducted according to the results using the following formula: Efficient rate = number of patients effectively treated/(number of patients that could not be evaluated) $\mathrm{x} 100 \%$.

Statistical analysis. Statistical analysis was performed using SAS software version 9.2 (SAS Institute, Cary, North Carolina, USA). Independent sample $t$-tests or Wilcoxon tests were used to compare continuous variables between groups, and paired t-test or Wilcoxon tests were used for comparison within groups. For the categorical variable analysis, a $\chi^{2}$ test or exact test were used for comparisons between groups. The Cochran-Mantel-Haenszel- $\chi^{2}$ test was used to analyze ordinal categorical data. Data were presented as the mean \pm standard deviation. $\mathrm{P}<0.05$ was considered to indicate a significantly significant result.

\section{Results}

Subjects. A total of 240 patients were originally eligible for this study, although the final full study group included 239 CRF subjects. The safety analysis set (SS) contained 236 patients, 118 in the experimental group and 118 in the control group.
The full analysis set (FAS) contained 225 patients, 109 in the experimental group and 116 in the control group. The per-protocol set (PPS) contained 203 patients, 95 in the experimental group and 108 in the control group.

Primary endpoint-neurotoxicity. As shown in Table I, in the FAS, at the 8th week of the study, ALC treatment reduced neurotoxicity in $50.5 \%$ of the experimental patients, compared with a $24.1 \%$ reduction in the control group. The difference between the ALC and placebo groups was statistically significant [95\% confidence interval (CI), 14.1-38.5\%; $\mathrm{P}<0.001]$. The 8 -week efficacy in the PPS was $51.6 \%$ (ALC) and $23.1 \%$ (control), which was significantly different (95\% CI, 15.6-41.2\%; P<0.001). The statistical significance of these findings was maintained following correction for the effect of different hospital centers $(\mathrm{P}<0.001$; data not shown). The reduction of neurotoxicity was time-dependent for the patients in both treatment groups. A comparison of the effect of ALC between the 4th, 8th, and 12th week revealed that the improvement in neurotoxicity was significantly different between the experimental and control group $(\mathrm{P}<0.05$; Table I). As shown in Table II, there was a observable improvement on the 4th week, with a decrease in the number of patients displaying grade $\geq 3$ neuropathy, and an increase in the number of patients displaying grade 2, although the differences between the two groups did not reach statistical significance $(\mathrm{P}>0.05)$. By the 8th week of treatment, the difference between the experimental and control group was statistical significant $(\mathrm{P}<0.05)$.

To further examine whether the efficacy of ALC was affected by chemotherapy treatment and cancer type, the cancer type in the treatment and placebo groups were classified prior to comparison of ALC efficacy. In the FAS, ALC was able to significantly improve the peripheral neuropathy grading in all cancer types, results which were not observed in the placebo group $(\mathrm{P}<0.05$; Fig. 1). ALC's effect was not affected by treatment with platinum-containing chemotherapy (Fig. 1). In addition, chemotherapy had no difference on the effect of ALC, compared with the (Fig. 2).

\section{Secondary endpoints}

Electrophysiological examination. Although the results from week 8 in the NCV test for the median nerve, ulnar nerve, common peroneal nerve, tibial nerve, superficial peroneal nerve and sural nerve demonstrate various degrees of improvement compared with the baseline at week 0 , only the NCV of the sural nerve was significantly different between the experimental and control groups $(\mathrm{P}<0.05$; data not shown).

Evaluation of electrophysiology. In the FAS, ALC treatment significantly improved $\mathrm{NCV}$ in the experimental group $(60.7 \%)$, as compared with the control group $(56.9 \%$; $\mathrm{P}<0.05)$. Similar results could be observed in the PPS group, with a $76.8 \%$ improvement in the experimental group and a $59.3 \%$ in the control group $(\mathrm{P}<0.05$; Table III).

Cancer-associated fatigue. In the FAS, ALC treatment reduced cancer-associated fatigue, however, the difference between the control (19.8\%) and treatment (31.2\%) groups was not statistically significant after 8 weeks treatment 
Table I. Peripheral sensory neurotoxicity evaluation at each visit at weeks 4, 8 and 12 .

\begin{tabular}{|c|c|c|c|c|c|c|c|c|}
\hline \multirow[b]{2}{*}{ Time } & \multicolumn{4}{|c|}{ Full analysis set } & \multicolumn{4}{|c|}{ Per-protocol set } \\
\hline & $\operatorname{ALC}(\%)$ & Placebo (\%) & $\chi^{2}$ & $\mathrm{P}^{\mathrm{a}}$ & $\operatorname{ALC}(\%)$ & Placebo (\%) & $\chi^{2}$ & $\mathrm{P}^{\mathrm{a}}$ \\
\hline \multicolumn{9}{|l|}{ Week 4} \\
\hline Valid & 29 (26.6) & $16(13.8)$ & 5.766 & 0.016 & $26(27.4)$ & $14(13.0)$ & 6.629 & 0.010 \\
\hline Invalid & $80(73.4)$ & $100(86.2)$ & & & $69(72.6)$ & $94(87.0)$ & & \\
\hline \multicolumn{9}{|l|}{ Week 8} \\
\hline Valid & $55(50.5)$ & $28(24.1)$ & 16.722 & $<0.001$ & 49 (51.6) & $25(23.1)$ & 17.636 & $<0.001$ \\
\hline Invalid & $54(49.1)$ & $88(75.9)$ & & & $46(48.4)$ & 83 (76.9) & & \\
\hline \multicolumn{9}{|l|}{ Week 12} \\
\hline Valid & $63(57.8)$ & 46 (39.7) & 7.406 & 0.007 & $57(60.0)$ & $41(38.0)$ & 9.830 & 0.002 \\
\hline Invalid & $46(42.2)$ & $70(60.3)$ & & & $38(40.0)$ & $67(62.0)$ & & \\
\hline
\end{tabular}

Neurotoxicities were graded according to National Cancer Institute Common Terminology Criteria for Adverse Events (version 3.0). Neurotoxicities were evaluated in patients before enrollment, and at weeks 4,8 and 12 . Neurotoxicity was defined as valid if the grade at weeks 4, 8 and 12 decreased compared with prior to enrollment. Otherwise, it was defined as invalid. ALC, acetyl-L-carnitine group; ${ }^{\text {PP-values were }}$ calculated with a two-sided $\chi^{2}$ test.

$(\mathrm{P}=0.0501)$. Conversely, in the PPS, the effect of ALC treatment was significantly different between the two groups at both week $8(\mathrm{P}<0.05)$ and $12(\mathrm{P}<0.05$; Table IV $)$.

KPS. In the FAS, ALC treatment induced a statistically significant improvement $(\mathrm{P}<0.05)$ in KPS $(29.3 \%)$ compared with the control group (13.0\%). In the PPS, the improvement rate was $31.6 \%$ in the experimental group, and $12.0 \%$ in the control group, a difference that was statistically significant $(\mathrm{P}<0.05)$. In both PPS and FAS, the improvement of KPS after 12 weeks of treatment was also statistically significant compared with the baseline $(\mathrm{P}<0.01)$.

Safety evaluation: Analysis of adverse reactions and events. Of the 236 patients, 41 reported a total of 62 incidents of adverse reactions. There was no significant difference in the number of adverse events between the experimental (19.5\%) and control (15.3\%) group ( $\mathrm{P}>0.05$; Table $\mathrm{V})$. The adverse reaction rate was $6.8 \%(8 / 118)$ for the trial group and $5.1 \%(6 / 118)$ for the control group, a difference that was not statistically significant $(\mathrm{P}>0.05$; Table $\mathrm{V})$. The primary adverse reactions were gastrointestinal reactions such as vomiting, abdominal distension and diarrhea. No statistically significant differences were observed between the treatment and control group $(\mathrm{P}>0.05)$. The most common adverse event was diarrhea [three cases in the ALC group (2.5\%) and two cases in the control group (1.7\%)]. Secondary events were decreased white blood cell count, liver dysfunction and insomnia. Notably, the three cases of decreased white blood cell count were all in the ALC group. There were three cases of liver dysfunction [one in the ALC group (0.8\%) and two in the control group (1.7\%)], and three cases of insomnia [two in the ALC group (1.7\%) and one in the control group (0.8\%)]. Four subjects in the ALC group withdrew from the study due to adverse events, but the adverse events in only one of these patients were associated with the drug. In total, seven severe adverse events (SAEs) occurred, two in the experimental group (1.7\%) and five in the control group (4.2\%). The incidence of adverse events was not statistically different between the treatment and control group $(\mathrm{P}>0.05)$, and none of the SAEs were associated with the drugs.

\section{Discussion}

Chemotherapy has a crucial role in the comprehensive treatment of cancer (14). However, CIPN is one of the most common dose-limiting adverse drug reactions (15-17). CIPN usually affects the dorsal root ganglia of primary sensory neurons. However, other sites, such as nerve terminals (distal terminations of the branches of an axon), may also be affected (14). Clinical features of CIPN vary depending on the type of agent involved and the site of action, and may include pure sensory or sensory-motor peripheral nerve damage of large myelinated or small unmyelinated fibers (14). The present study evaluated the efficacy and safety of ALC in Chinese patients with CIPN.

In this clinical trial, the therapeutic effect of ALC on neurotoxicity became evident after 8 weeks of treatment, and neuropathy was significantly reduced in patients treated with ALC. However, improvement of CIPN was a slow process, and statistically significant differences were not observed until week 8 after treatment. At week 12, there remained a significant difference between the ALC and the placebo group, demonstrating that the improvement in neurotoxicity persists without further clinical intervention (including discontinuation of chemotherapeutics).

Electrophysiological tests of neuronal function revealed statistical differences between the experimental and control group, with improvement of electrophysiological function after 8 weeks of treatment. These findings indicate that ALC is able to improve neuronal function in patients following chemotherapy, consistent with previous studies which demonstrated that prophylactic administration of oral ALC prevents the development of paclitaxel-induced painful peripheral neuropathy $(18,19)$. Nerve conduction velocity, one of the objective 
Table II. Peripheral sensory neurotoxicity grading.

\begin{tabular}{|c|c|c|c|c|}
\hline CTC grade & $\operatorname{ALC}(\%)$ & Placebo (\%) & $\chi^{2}$ & $\mathrm{P}^{\mathrm{a}}$ \\
\hline \multicolumn{5}{|l|}{ FAS } \\
\hline \multicolumn{5}{|l|}{ Baseline } \\
\hline I & $0(0.0)$ & $0(0.0)$ & 1.175 & 0.278 \\
\hline II & $42(38.5)$ & $53(45.7)$ & & \\
\hline III & $67(61.5)$ & $63(54.3)$ & & \\
\hline \multicolumn{5}{|l|}{ 4th week } \\
\hline I & $6(5.6)$ & $8(7.3)$ & 0.873 & 0.646 \\
\hline II & $61(57.0)$ & $56(50.9)$ & & \\
\hline III & $40(37.4)$ & $46(41.8)$ & & \\
\hline \multicolumn{5}{|l|}{ 8th week } \\
\hline I & $27(25.2)$ & $20(18.2)$ & 6.242 & 0.0441 \\
\hline II & $54(50.5)$ & $46(41.8)$ & & \\
\hline III & $26(24.3)$ & $44(40.0)$ & & \\
\hline \multicolumn{5}{|l|}{ 12th week } \\
\hline I & 37 (34.6) & $28(25.5)$ & 3.7594 & 0.153 \\
\hline II & 48 (44.9) & $48(43.6)$ & & \\
\hline III & $22(20.6)$ & 34 (30.9) & & \\
\hline \multicolumn{5}{|l|}{ PPS } \\
\hline \multicolumn{5}{|l|}{ Baseline } \\
\hline I & $0(0.0)$ & $0(0.0)$ & $1.910^{\mathrm{b}}$ & 0.167 \\
\hline II & $34(35.8)$ & $49(45.4)$ & & \\
\hline III & $61(64.2)$ & $59(54.6)$ & & \\
\hline \multicolumn{5}{|l|}{ 4th week } \\
\hline I & $5(5.3)$ & $8(7.8)$ & $1.007^{\mathrm{b}}$ & 0.605 \\
\hline II & $52(55.3)$ & $50(49.0)$ & & \\
\hline III & $37(39.4)$ & $44(43.1)$ & & \\
\hline \multicolumn{5}{|l|}{ 8th week } \\
\hline I & $22(23.4)$ & 18 (17.6) & $5.364^{\mathrm{b}}$ & 0.068 \\
\hline II & $48(51.1)$ & $42(41.2)$ & & \\
\hline III & $24(25.5)$ & $42(41.2)$ & & \\
\hline \multicolumn{5}{|l|}{12 th week } \\
\hline I & $32(34.0)$ & $25(24.5)$ & $4.299^{\mathrm{b}}$ & 0.117 \\
\hline II & $43(45.7)$ & $44(43.1)$ & & \\
\hline III & $19(20.2)$ & $33(32.4)$ & & \\
\hline
\end{tabular}

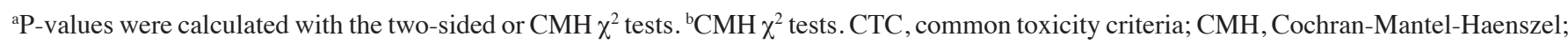
ALC, acetyl-L-carnitine; FAS, full analysis set; PPS, per-protocol set.

indicators of peripheral neuropathy was also improved, but this improvement was less marked than the improvement in the subjective symptoms of the patients. Comparison of the neuronal conduction velocity between the baseline and week 8 of the trial indicated that only the sural sensory nerve exhibited a significant improvement in function. The following factors may be responsible: i) Motor neuron toxicity appears in the early stages of chemotherapy, thus, following timely treatment, neuronal function was improved and the difference in conduction speed of the two groups of motor neurons was not statistically significant; ii) the median, ulnar, and superficial peroneal nerves are composed of finer fibers and are located peripherally compared with the nervus suralis neural fiber of the leg, and the coarser fibers are thus more resistant to CIPN; iii) the sensory nerve fibers recover more rapidly, and therefore only the sural sensory nerve demonstrated significant treatment differences.

At the 4th week of this study, the number of patients with grade 3 decreased, but there was no statistical difference between the two groups. By the 8 th week, the difference between the two groups gained statistical significance, suggesting that the onset and process of CIPN improvement is slow. Therefore, the treatment of CIPN requires long-term medication. After 4 weeks of ALC withdrawal (12th week of 
Table III. Nerve electrophysiological function (Week 8).

\begin{tabular}{|c|c|c|c|c|c|c|c|c|}
\hline \multirow[b]{2}{*}{ Effect } & \multicolumn{4}{|c|}{ Full analysis set } & \multicolumn{4}{|c|}{ Per-protocol set } \\
\hline & $\operatorname{ALC}(\%)$ & Placebo (\%) & $\chi^{2}$ & $\mathrm{P}^{\mathrm{a}}$ & $\operatorname{ALC}(\%)$ & Placebo (\%) & $\chi^{2}$ & $\mathrm{P}^{\mathrm{a}}$ \\
\hline Marked effect & $22(20.2)$ & $17(14.7)$ & 4.133 & 0.126 & $21(22.1)$ & $16(14.8)$ & 7.330 & 0.026 \\
\hline Improved & $54(49.5)$ & $49(42.2)$ & & & $52(54.7)$ & $48(44.4)$ & & \\
\hline Unchanged & $33(30.3)$ & $50(43.1)$ & & & $22(23.2)$ & $44(40.7)$ & & \\
\hline Valid & $76(60.7)$ & $66(56.90)$ & 3.972 & 0.046 & $73(76.8)$ & $64(59.3)$ & 7.121 & 0.008 \\
\hline Invalid & & & & & $22(23.2)$ & $44(40.7)$ & & \\
\hline
\end{tabular}

Valid, marked effect + improved; invalid, unchanged. A marked effect was defined if the decreased rate was $\geq 50 \%$. Improved was defined if the decreased rate was $\geq 16 \%$ and $<50 \%$. Unchanged was defined if the decreased rate was $<16 \%$. ${ }^{\text {P }}$-values were calculated with a two-sided $\chi^{2}$ test. ALC, acetyl-L-carnitine.

Table IV. Cancer-associated fatigue.

\begin{tabular}{|c|c|c|c|c|}
\hline CTC grade & $\operatorname{ALC}(\%)$ & Placebo (\%) & $\chi^{2}$ & $\mathrm{P}$ \\
\hline \multicolumn{5}{|l|}{ FAS } \\
\hline \multicolumn{5}{|l|}{ 4th week } \\
\hline Valid & 17 (15.6) & $16(13.8)$ & 0.146 & 0.702 \\
\hline Invalid & $92(84.4)$ & $100(86.2)$ & & \\
\hline \multicolumn{5}{|l|}{ 8th week } \\
\hline Valid & $34(31.2)$ & $23(19.8)$ & 3.837 & 0.050 \\
\hline Invalid & $75(68.8)$ & $93(80.2)$ & & \\
\hline \multicolumn{5}{|l|}{ 12th week } \\
\hline Valid & $41(37.6)$ & $31(26.7)$ & 3.063 & 0.080 \\
\hline Invalid & $68(62.4)$ & $85(73.3)$ & & \\
\hline \multicolumn{5}{|l|}{ PPS } \\
\hline \multicolumn{5}{|l|}{ 4th week } \\
\hline Valid & 17 (17.9) & $14(13.0)$ & 0.950 & 0.330 \\
\hline Invalid & $78(82.1)$ & $94(87.0)$ & & \\
\hline \multicolumn{5}{|l|}{ 8th week } \\
\hline Valid & $32(33.7)$ & $20(18.5)$ & 6.100 & 0.014 \\
\hline Invalid & $63(66.3)$ & $88(81.5)$ & & \\
\hline \multicolumn{5}{|l|}{ 12th week } \\
\hline Valid & $39(41.1)$ & $27(25.0)$ & 5.936 & 0.015 \\
\hline Invalid & $56(58.9)$ & $81(75.0)$ & & \\
\hline
\end{tabular}

FAS, full analysis set; PPS, per-protocol set; ALC, acetyl-L-carnitine. P-values were calculated with a two sided $\chi^{2}$ test.

trial), the two groups returned to being statistically indistinguishable from one another. The trial group improved more than the control group with 37 grade 1 patients $(34.6 \%)$, compared with $28(25.5 \%)$ in the control group, and 22 cases $(20.6 \%)$ of grade 3 patients in the trial group, compared with 34 cases $(30.9 \%)$ in the control group. It was hypothesized that if this treatment period was extended to 12 weeks, the improvement would be further increased.

In the current study, the efficacy of ALC on CIPN improvement was not associated with the subject cancer types or the chemotherapy. Although chemotherapy may induce CIPN via various pathways, the main characteristics are inflammation and neuronal necrosis. Given the potential influence of chemotherapy, all patients in the study had not previously received chemotherapy (20-23). Another important point is all the subjects in the study has stopped and would not receive the chemotherapy. Therefore, the influence of chemotherapy may be eliminated.

In the PPS, the ALC group had significantly diminished cancer-associated fatigue compared with the control group. In both the FAS and PPS, the ALC group exhibited significant improvements in KPS compared with the control group. These 
Table V. Comparison of adverse reaction and adverse event incidence.

\begin{tabular}{lccr}
\hline Incident & ALC (\%) & Placebo (\%) & $\chi^{2}$ \\
\hline Adverse events & & & 0.738 \\
Yes & $23(19.5)$ & $18(15.3)$ & 0.3903 \\
No & $95(80.5)$ & $100(84.7)$ & 0.3037 \\
Adverse reaction & $8(6.8)$ & $6(5.1)$ & 0.5816 \\
Yes & $110(93.2)$ & $112(94.9)$ & \\
No & & & \\
\hline
\end{tabular}

ALC, acetyl-L-carnitine; ${ }^{\text {a }}$-values were calculated with a two sided $\chi^{2}$ test.
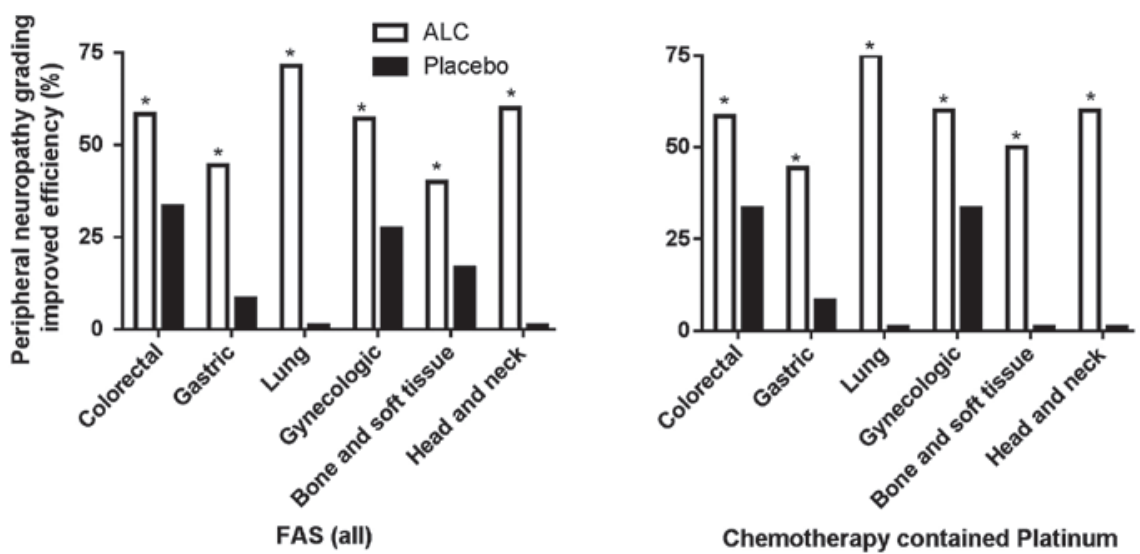

Figure 1. Peripheral neuropathy grading improved efficiency in patients with various types of cancer. Cancer types of the patients enrolled in the study include colorectal, gastric, lung, gynecological, bone and soft tissue, and head and neck cancers. Peripheral neuropathy grading improved efficiency was measured in the patients receiving either ALC or placebo. ${ }^{*} \mathrm{P}<0.05$ vs. placebo. ALC, acetyl-L-carnitine; FAS, full analysis set.
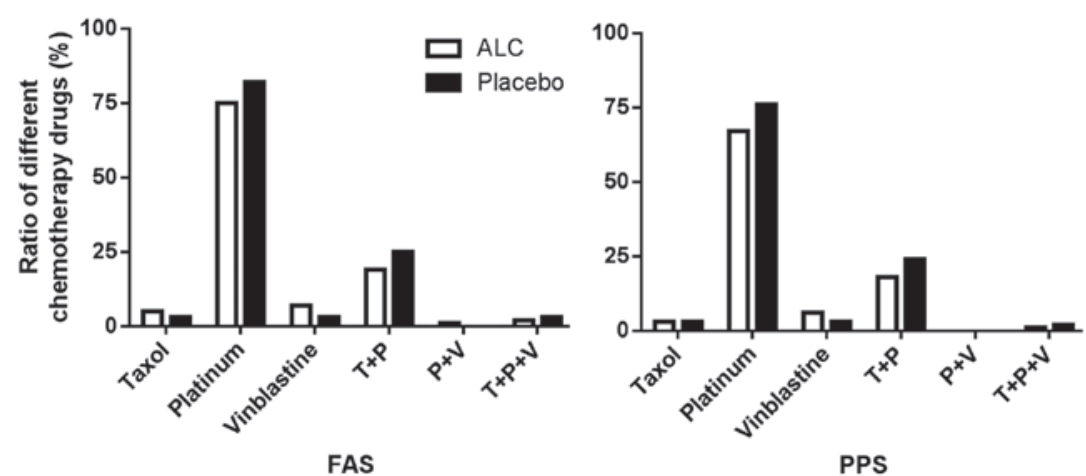

Figure 2. Ratio of various chemotherapy drugs. Chemotherapy drugs used in the patients receiving either ALC or placebo, including taxol, platinum and vinblastine. FAS, full analysis set; PPS, per-protocol set; ALC, acetyl-L-carnitine.

results demonstrate that ALC is able to ease cancer-associated fatigue and improve the physical condition of patients following chemotherapy. Fatigue, caused by tumors and their associated treatment is a common problem for patients with cancer, and chronic fatigue seriously diminishes patient quality of life. Currently, there is a lack of pharmacological therapies for the treatment of cancer-associated fatigue. The results of this clinical study demonstrate that ALC is able to reduce cancer-associated fatigue, thus improving patient quality of life.

The overall incidence of adverse reactions in this clinical study was $5.9 \%$, with $6.8 \%$ for the ALC group. In total, $3.4 \%$ of the patients withdrew from the study due to adverse reactions. These results demonstrate that ALC is well tolerated in patients, which is consistent with previous studies (24-27). Seven cases of SAE [two in the trial group (1.7\%) and five in the control group (4.2\%)] were recorded, although none of the SAEs were determined to be associated with drug administration. These findings provide evidence of the high safety profile of this type of treatment intervention.

In conclusion, to the best of our knowledge, the present study provides the first demonstration of the efficacy and safety of ALC for reducing chemotherapy-induced peripheral 
neuropathy toxicity and its associated symptoms. Based on these results, ALC may have an important role in the treatment of chemotherapy-induced peripheral neuropathy in China.

\section{Acknowledgements}

The authors of the present study would like to thank Professor Zhao Naiqing and Professor Luo Jiangfeng for their advice and help with the statistical analysis involved in the present study, and are grateful to Dr. Yao Chaoya and Dr. Qian Xuemei for their participation with the electrophysiology examination. This study was funded by Lee's Pharmaceutical (Hong Kong) Ltd. and Sigma-Tau Pharmaceutical, Inc., (Pomezia, Italy).

\section{References}

1. Ocean AJ and Vahdat LT: Chemotherapy-induced peripheral neuropathy: Pathogenesis and emerging therapies. Support Care Cancer 12: 619-625, 2004.

2. Urba SG, Orringer MB, Ianettonni M, Hayman JA and Satoru H: Concurrent cisplatin, paclitaxel and radiotherapy as preoperative treatment for patients with locoregional esophageal carcinoma. Cancer 98: 2177-2183, 2003.

3. Brewer JR, Morrison G, Dolan ME and Fleming GF: Chemotherapy-induced peripheral neuropathy: Current status and progress. Gynecol Oncol 140: 176-183, 2016

4. Taglialatela G, Angelucci L, Ramacci MT, Werrbach-Perez K, Jackson GR and Perez-Polo JR: Stimulation of nerve growth factor receptors in PC12 by acetyl-L-carnitine. Biochem Pharmacol 44: 577-585, 1992.

5. Westlund KN, Lu Y, Werrbach-Perez K, Hulsebosch CE, Morgan B, Pizzo DP, Eisenberg HM and Perez-Polo JR: Effects of nerve growth factor and acetyl-L-carnitine arginyl amide on the human neuronal line HCN-1A. Int J Dev Neurosci 10: 361-373, 1992.

6. Pisano C, Pratesi G, Laccabue D, Zunino F, Lo Giudice P, Bellucci A, Pacifici L, Camerini B, Vesci L, Castorina M, et al: Paclitaxel and Cisplatin-induced neurotoxicity: A protective role of acetyl-L-carnitine. Clin Cancer Res 9: 5756-5767, 2003.

7. Ghirardi O, Lo Giudice P, Pisano C, Vertechy M, Bellucci A, Vesci L, Cundari S, Miloso M, Rigamonti LM, Nicolini G, et al: Acetyl-L-Carnitine prevents and reverts experimental chronic neurotoxicity induced by oxaliplatin, without altering its antitumor properties. Anticancer Res 25: 2681-2687, 2005.

8. Xiao WH and Bennett GJ: Chemotherapy-evoked neuropathic pain: Abnormal spontaneous discharge in A-fiber and C-fiber primary afferent neurons and its suppression by acetyl-L-carnitine. Pain 135: 262-270, 2008.

9. Argyriou AA, Velasco R, Briani C, Cavaletti G, Bruna J, Alberti P, Cacciavillani M, Lonardi S, Santos C, Cortinovis D, et al: Peripheral neurotoxicity of oxaliplatin in combination with 5-fluorouracil (FOLFOX) or capecitabine (XELOX): A prospective evaluation of 150 colorectal cancer patients. Ann Oncol 23: 3116-3122, 2012

10. Portenoy RK and Itri LM: Cancer-related fatigue: Guidelines for evaluation and management. Oncologist 4: 1-10, 1999.
11. de Kock I, Mirhosseini M, Lau F, Thai V, Downing M, Quan H, Lesperance M and Yang J: Conversion of Karnofsky Performance Status (KPS) and Eastern Cooperative Oncology Group Performance Status (ECOG) to Palliative Performance Scale (PPS), and the interchangeability of PPS and KPS in prognostic tools. J Palliat Care 29: 163-169, 2013.

12. Iwasaki N, Ogata M, Tomonaga O, Kuroki H, Kasahara T, Yano $\mathrm{N}$ and Iwamoto $\mathrm{Y}$ : Liver and kidney function in Japanese patients with maturity-onset diabetes of the young. Diabetes Care $21: 2144-2148,1998$.

13. Fisman EZ, Motro M, Tenenbaum A, Boyko V, Mandelzweig L and Behar S: Impaired fasting glucose concentrations in nondiabetic patients with ischemic heart disease: A marker for a worse prognosis. Am Heart J 141: 485-490, 2001.

14. Argyriou AA, Kyritsis AP, Makatsoris T and Kalofonos HP: Chemotherapy-induced peripheral neuropathy in adults: A comprehensive update of the literature. Cancer Manag Res 6: 135-147, 2014.

15. Brzezinski K: Chemotherapy-induced polyneuropathy. Part I. Pathophysiology. Contemp Oncol (Pozn) 16: 72-78, 2012.

16. Carozzi VA, Canta A and Chiorazzi A: Chemotherapy-induced peripheral neuropathy: What do we know about mechanisms? Neurosci Lett 596: 90-107, 2015.

17. Mols F, Beijers T, Vreugdenhil G and van de Poll-Franse L: Chemotherapy-induced peripheral neuropathy and its association with quality of life: A systematic review. Support Care Cancer 22: 2261-2269, 2014.

18. Flatters SJ, Xiao WH and Bennett GJ: Acetyl-L-carnitine prevents and reduces paclitaxel-induced painful peripheral neuropathy. Neurosci Lett 397: 219-223, 2006.

19. Jin HW, Flatters SJ, Xiao WH, Mulhern HL and Bennett GJ: Prevention of paclitaxel-evoked painful peripheral neuropathy by acetyl-L-carnitine: Effects on axonal mitochondria, sensory nerve fiber terminal arbors, and cutaneous Langerhans cells. Exp Neurol 210: 229-237, 2008 .

20. Scholz J and Woolf CJ: The neuropathic pain triad: Neurons, immune cells and glia. Nat Neurosci 10: 1361-1368, 2007.

21. Marchand F, Perretti M and McMahon SB: Role of the immune system in chronic pain. Nat Rev Neurosci 6: 521-532, 2005.

22. Uceyler N, Kafke W, Riediger N, He L, Necula G, Toyka KV and Sommer C: Elevated proinflammatory cytokine expression in affected skin in small fiber neuropathy. Neurology 74: 1806-1813, 2010.

23. Areti A, Yerra VG, Naidu V and Kumar A: Oxidative stress and nerve damage: Role in chemotherapy induced peripheral neuropathy. Redox Biol 2: 289-295, 2014.

24. Malaguarnera M, Gargante MP, Cristaldi E, Colonna V, Messano M, Koverech A, Neri S, Vacante M, Cammalleri L and Motta M: Acetyl L-carnitine (ALC) treatment in elderly patients with fatigue. Arch Gerontol Geriatr 46: 181-190, 2008.

25. Maestri A, De Pasquale Ceratti A, Cundari S, Zanna C, Cortesi E and Crinò L: A pilot study on the effect of acetyl-L-carnitine in paclitaxel- and cisplatin-induced peripheral neuropathy. Tumori 91: 135-138, 2005.

26. Evans JD, Jacobs TF and Evans EW: Role of acetyl-L-carnitine in the treatment of diabetic peripheral neuropathy. Ann Pharmacother 42: 1686-1691, 2008.

27. Bianchi G, Vitali G, Caraceni A, Ravaglia S, Capri G, Cundari S, Zanna C and Gianni L: Symptomatic and neurophysiological responses of paclitaxel- or cisplatin-induced neuropathy to oral acetyl-L-carnitine. Eur J Cancer 41: 1746-1750, 2005. 